\begin{tabular}{|c|c|c|c|}
\hline Eiszeitalter u. Gegenwart & 39 & $\begin{array}{c}1-9 \\
4 \mathrm{Abb} .\end{array}$ & Hannover 1989 \\
\hline
\end{tabular}

\title{
Neue Erkenntnisse zur Entstehung pleistozäner Talverfüllungen
}

\author{
Michael BeCHT *)
}

\author{
Valley sediments, Pleistocene, moraines, \\ glaciolacustrine sedimentation, influence, glaciation, fluvial erosion \\ Bavaria, Alpenvorland
}

Kurzfassung: Während des Pleistozäns wurden in Seitentälern der nördlichen Kalkvoralpen mächtige Lockersedimente abgelagert. Die Gletscher stießen dabei von den Haupttälern in die Seitentäler vor und schufen dort Sedimentfallen.

Die Moränensedimente im Lainbachtal bei Benediktbeuern wurden bisher als Staubeckensedimente angesprochen, die in einen Stausee sedimentiert wurden. Anhand des Vergleiches der spät- und postglazialen Ausräummasse mit der Menge akkumulierter Sedimente in dem Schwemmfächer des Lainbaches konnte diese Vorstellung widerlegt werden. Weitere Untersuchungen zum Rundungsgrad und Kristallingehalt von Geröllen und Auftreten von Schichtung im Lockersediment belegen, daß hier Moränenablagerungen des Ferneises vorliegen.

Durch hochglazialen Gletschervorstoß sowie zahlreiche Gletscherstandsschwankungen während des Rückschmelzens wurden im Lainbachtal Verebnungen geschaffen, die heute noch in Resten erhalten sind.

Der Verlauf fluvialer Erosion seit dem Spätglazial ist noch nicht ausreichend bekannt. Eine Verstärkung der Abtragung mit den Eingriffen des Menschen in den Naturhaushalt vor allem seit dem Mittelalter scheint dagegen gut belegt.

\section{[New Researches about the Genesis of Pleistocene Loose Sediments in Small Alpine Side Valleys]}

Abstract: In the course of the Pleistocene, thick loose sediments were deposited in side valleys of the limestone Alps. The glaciers advanced from the main valleys into the side valleys and shaped them into sedimentary basins.

The morainic deposit in the Lainbach valley near Benediktbeuern has so far been explained as a sediment which had been deposited in a glacially dammed lake.

But a comparision of the mass of the late - to post glacial eroded sediment with the mass of the accumulated loose sediments in the alluvial cone of the Lainbach shows that this

*) Anschrift des Verfassers: Dr. M. BECHT, Institut für Geographie der Universität München, Luisenstraße 37, D -8000 München 2 . assumption is not right. Further studies on the degree of roundness, the content crystalline, and the stratification of the loose sediment prove the existence of a moraine deposit in the Lainbach valley. The glacial advance in the high glacial and many glacier oscillations during the period of recession created planation surface. Relics of these surfaces can still be found today.

The progress of the fluvial erosion since the late glacial period is still not known in detail. An increase of the fluvial erosion due to human activities in the forest of Lainbach catchment, especially since the middle ages, is well evidenced.

\section{Einleitung}

Die Bezeichnung ,pleistozäne Talverbauung” wird für Lockersedimente gebraucht, die vor allem während der Würm- aber auch in der Riß- und Mindeleiszeit (FRENZEL 1987) in alpinen Seitentälern abgelagert wurden. Das vorher entwickelte Talrelief ist verschüttet worden; ihm folgen die sich postglazial einschneidenden Ablußsysteme nur zum Teil wieder (BADER 1985). Glazialepigenetische Durchbrüche sind hierauf zurückzuführen. Die Ablagerung der Lockersedimente während des Pleistozäns ist auf zweifache Weise vorstellbar:

1. Fluvialer Transport erodierten Materials in einen Stauraum, der durch den im Haupttal liegenden und den Ausgang des Nebentales versperrenden Gletscher entstand.

2. Glazialer Transport durch den vom Haupttal in die Seitentäler vorstoßenden Gletscher.

Die gebräuchliche Bezeichnung „Talverbauung” bezieht sich auf die Stauraumtheorie. Diese Festlegung (KARL \& DANZ 1969; DOBEN 1985) hat nun weitreichende Konsequenzen hinsichtlich der Berechnung des Ausraumvolumens und der Einschätzung der aktuellen Erosionsleistung im Vergleich zu der spätglazialen und frühholozänen Abtragung, da man mit der Vorstellung eines Stauraumes zu erheblich 
größeren Erosionsbeträgen kommen muß als bei der Mächtigkeit eines durch Gletschereis weitgehend ausgefüllten Seitentales.

Die Reste dieser pleistozänen Lockersedimente, die im deutschen Alpenraum in nahezu jedem Seitental von Iller, Lech, Isar, Loisach und Inn in unterschiedlichen Mächtigkeiten erhalten sind, gehören zu den am stärksten erosionsgefährdeten Bereichen dieser Landschaftseinheit. Aus Erosionsanbrüchen werden den Vorflutern große Mengen Feststoffe zugeführt.

Im Folgenden sollen die Entstehung einer pleistozänen Talverfüllung und die sich spät- und postglazial anschließende Erosion des Sedimentkörpers dargestellt werden. Es wurde dazu das Lainbachtal bei Benediktbeuern ausgewählt, ein Seitental der Loisach, da hier schon früher Arbeiten zu diesen Fragen angefertigt wurden (KARL \& DANZ 1969) und seit einigen Jahren intensiv Messungen zur aktuellen und historischen Erosionsleistung durchgeführt werden (BRECHT 1986; BECHT \& KoPP 1988).

\section{Die Genese der Lockersedimente im Lainbachtal}

\subsection{Vorstellungen zur Staubeckensedimentation}

Während in älteren Arbeiten der Aufbau und die Verbreitung pleistozäner Ablagerungen im Lainbachgebiet recht differenziert dargestellt ist (MÜLLER-DEILE 1940), wird in neueren Veröffentlichungen die Vorstellung vertreten, daß sich in den „Seitentälern Eisrandseen bilden konnten" (KARL \& DANZ 1969: 17), in denen sich "Stauseeablagerungen" (DOBEN 1985: 43) absetzten. „Dieser seitliche Aufstau (sei) eine verbreitete Erscheinung am Alpenrand" (KARL \& DANZ 1969: 82). Die Autoren gehen davon aus, daß „die Stauschotter (...) kurze Zeit unter Eis begraben" (KARL DANZ 1969: 82) wurden, als das Ferneis seine maximale Mächtigkeit erreicht hatte und nun erst in das Tal vorstieß. Das überdeckende Ferneis soll während des Würmmaximums nicht mächtiger als 40-80 m gewesen sein.

Schon der bloße Augenschein der in zahlreichen großen Erosionskerben gut aufgeschlossenen Lockersedimente läßt an dieser Vorstellung zweifeln. Eine $\mathrm{S}$ c h i c h t u n g des Materials und eine bei der Ablagerung eintretende Korngrößensortierung ist nur an einigen Stellen an der Oberkante der Talverfüllung nachweisbar.

$\mathrm{B}$ ä $\mathrm{nder}$ t o n e an der Basis der Ablagerungen werden zwar oft postuliert (KARL \& DANZ 1969), sind aber nicht im Gelände nachweisbar. Lediglich in 760 $m$ ü. NN finden sich an den Hängen im Unterlauf des Lainbaches tonig-schluffige Ablagerungen, die jedoch nicht als Basistone angesprochen werden können (vgl. 2.4.). Geht man von der Ablagerung in einen großen Stauraum aus, so muß das Sediment auch fluvial transportiert worden sein und damit gegenüber Moränenmaterial gerundeter erscheinen. Die G e s c h i e b e z ä h l u n g e n von MuXFeLDT (1972) belegen, daß zwischen dem Wallmoränenmaterial und den Stausedimenten, die an fünf Stellen im Lainbachgebiet untersucht wurden, keine signifikanten Unterschiede bestehen.

In den Lockersedimenten der Talverfüllung sind im gesamten Untersuchungsgebiet $\mathrm{K}$ r is t a $11 \mathrm{in}$ g e s c h i e b e aus den Zentralalpen nachweisbar. Auch diese Tatsache spricht gegen die Stauraumtheorie, da sich in einem solchen Becken überwiegend der lokale Verwitterungsschutt ansammeln würde, während der Kristallinanteil im Geschiebe die Beteiligung des Ferneises belegt.

Die $M$ ächtigkeit d e r M o r än e n wäl$1 \mathrm{e}$ mit relativen Höhen bis zu $40 \mathrm{~m}$ am Gurnberg ist mit der Vorstellung einer kurzzeitigen, getingen Eisbedeckung (KARL \& DANZ 1969) während des Würmmaximums schwer vereinbar. Ein Felskern in diesen Moränen ist nach den Ergebnissen neuerer geoseismischer Untersuchungen auszuschließen. Die Moränenwälle sind Sedimenten der Talverfüllung aufgesetzt, die wesentlich dichter als erstere lagern und außergewöhnlich hohe seismische Geschwindigkeiten (bis zu $3.1 \mathrm{~km} / \mathrm{s}$ in tiefen Lagen) aufweisen (BADER 1985). Diese hohe Geschwindigkeiten lassen auf eine stärkere Eisbedeckung des Sedimentkörpers schließen, als bisher angenommen wurde (BADER 1979).

Aufgrund ihrer Vorstellung eines mit Sedimenten vollkommen verfüllten Tales berechnen KARL \& DANZ (1969: 17) die A u s r a u m m s s e. Sie gehen im Lainbachtal von einer „Oberfläche des Eisrandsees” von $5.6 \mathrm{qkm}$ aus und ermitteln 530 Mio cbm erodiertes Material. Nach eigenen Berechnungen müßte die Ausraummasse sogar 746 Mio cbm betragen. Wie die von KARL \& DANZ konstruierte Ausraumkurve für das Halblechgebiet (Abb. 1) zeigt, soll die höchste Austragsintensität im frühen Holozän stattgefunden haben. Damit müssen auch die Schwemmfächer, die die Wildbäche der Seitentäler in die Haupttäler schütten, im Holozän aufgebaut worden sein. Es liegt daher nahe, das Volumen der Ausraummasse mit dem Inhalt des Schotterkörpers zu vergleichen. Auf dem $\mathrm{Schwe} m \mathrm{mfäch}$ er des La in b a h e s wurden bisher lediglich drei Bohrungen bis zum anstehenden Fels abgeteuft, so daß eine flächenhafte Auswertung nicht möglich ist. Zusätzliche Hinweise erhält man allerdings durch den besser erschlossenen Schwemmfächer des nördlich anschließenden Steinbaches. Aus den Unterlagen der Bohrungen, die im Bohrarchiv des Bayerischen Geologischen Landes- 


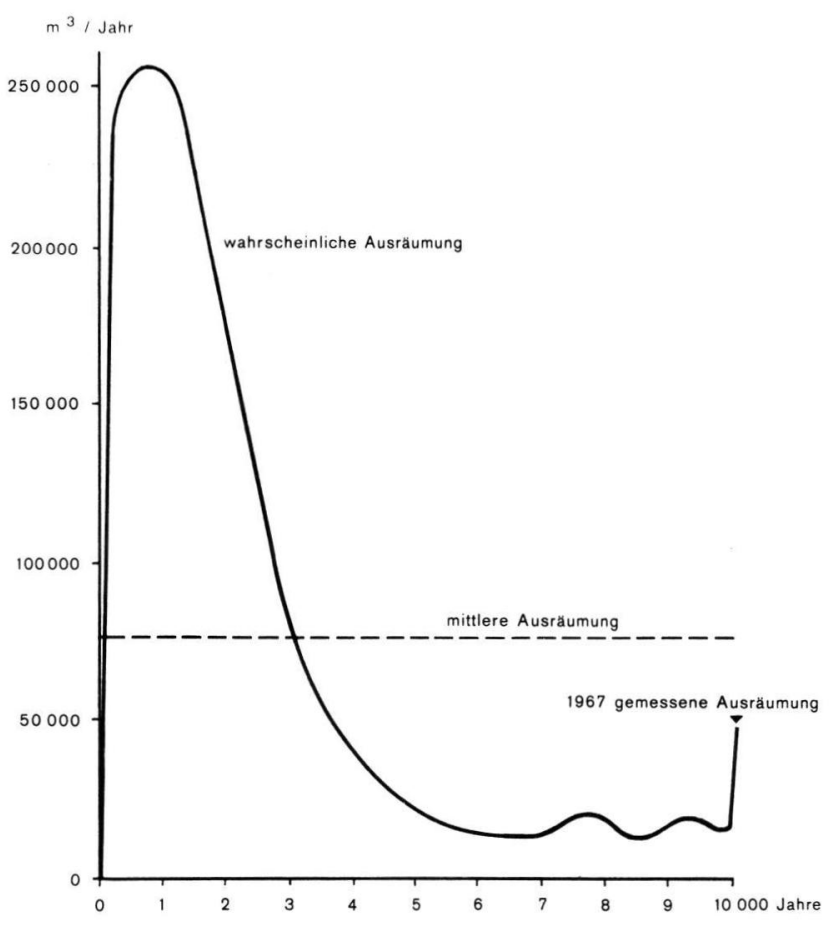

Abb. 1: Mittlere und wahrscheinliche Ausräumung der Talverfüllung des Halblechs in den letzten 10000 Jahren (KARL \& DANZ 1969: 21).

The average and the probable rate of erosion of the pleistocene loose sediments in the Halblech valley since $10000 \mathrm{bp}$. amtes eingesehen werden konnten, ergibt sich eine Schottermächtigkeit von durchschnittlich $8-10 \mathrm{~m}$. Bei einer Fläche des Schwemmfächers des Lainbaches von $12.25 \mathrm{qkm}$ ergibt sich daraus ein Volumen von 98-122 Mio cbm Schotter. Da die Schottermächtigkeit nach Westen ausdünnt, liegt dieser Wert, der nur $15 \%$ der angenommenen Ausraummasse umfaßt, an der oberen Grenze. Weite Teile des Schwemmfächers unterhalb Benediktbeuerns werden von mächtigen Quellmooren gebildet, wie sich durch Bohrungen, die hier gemeinsam mit FRENZEL (Universität Hohenheim) niedergebracht wurden, zeigte (FRENZEL 1987). Diese Moore bilden sich auf den schluffig-tonigen Ablagerungen im Kochelseebecken und täuschen morphologisch einen Schwemmfächer nur vor.

Ein Teil der Lockersedimente ist sicherlich mit den Abflüssen aus dem Tal in den Vorfluter transportiert und somit nicht auf dem Schwemmfächer abgelagert worden. Aber selbst unter der Annahme, daß die Ton- und Schlufffraktion nicht abgelagert wurde, befinden sich im Lainbachschwemmfächer nur höchstens $20 \%$ der Sedimente, die aus dem Tal ausgeräumt sein müßten. Aus dem Vergleich der erodierten und sedimentierten Schotter läßt sich die Stauseevorstellung ebenfalls nicht belegen.

Nach der dargelegten Argumentation muß die Vorstellung eines großen Stauraumes, der mit der Abdämmung des Lainbachtales durch den Isar-Walchen- see- und den Loisachgletscher entstand, verworfen werden. Beide Ferngletscher trafen im Kochelseebecken zusammen. Eine Eismasse, die mehrere hundert Meter mächtig war, konnte sich als plastisch reagierender Körper auch in das Seitental (Lainbachtal) vorschieben. In der Stauraumvorstellung bleibt unverständlich, weshalb das Ferneis lange Zeit vor dem Taleingang verweilte und sich erst später in geringer Mächtigkeit auf die schon gebildete Talverfüllung aufschob, um dann noch hohe Moränenwälle im Tal zu schaffen.

\subsection{Entstehung der Lockersedimente durch Ferngletscher im Lainbachtal}

Mit dem Vorstoß des Isar-Walchensee- und des Loisachgletschers im Würmglazial wurde auch das Lainbachtal mit Ferneis erfüllt. Ein Zustrom aus dem Isartal über die östliche Wasserscheide an der Sattelalm (1092 $\mathrm{m}$ ü. NN) ist auszuschließen, da die Oberkante des Moränenmaterials in diesem Bereich bei $1050 \mathrm{~m}$ ü. NN liegt. Abbildung 2 zeigt die Lage der F e r $\mathrm{n}$ m o r ä n e n w ä 11 e, die im Tal nachweisbar sind. Die vermutete ehemalige Lage der jetzt erodierten Teile der Wälle wurden gestrichelt hinzugefügt. Die Unterscheidung der Moränen des Ferneises von denjenigen der Lokalvergletscherung, die in den Karen am Fuße der Benedikten- und Glaswand bestand, erfolgt eindeutig über den Gehalt an Kristallingeschieben. 


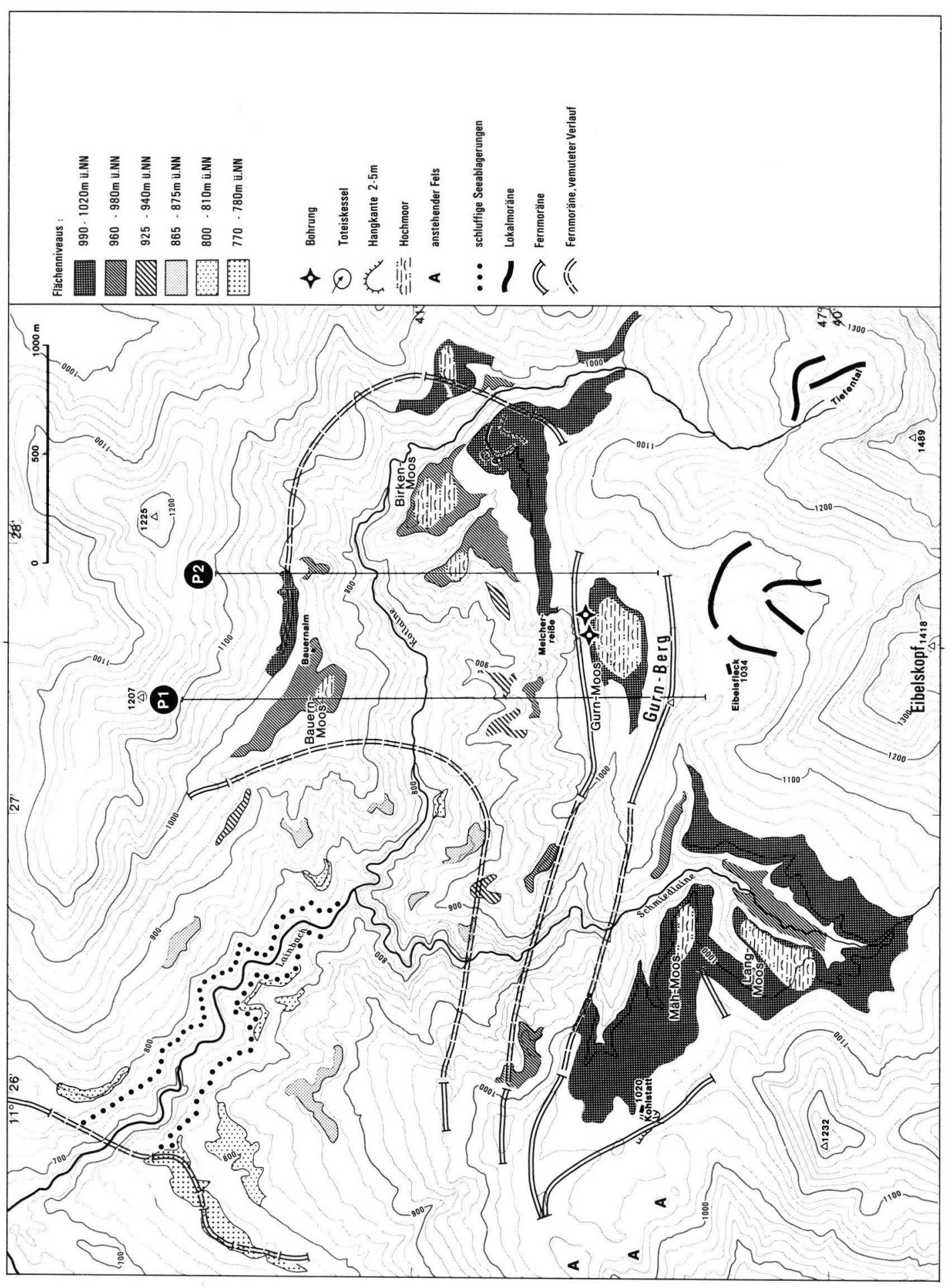

Abb. 2: Verebnungen und Moränenwalle im Lainbachgebiet.

Fig. 2: Planation surfaces and moraine dams in the Lainbach valley. 
Die Fernmoränenwälle weisen einen in Abhängigkeit von ihrer Lage im Tal unterschiedlichen Gehalt kristalliner Geschiebe auf. Während auf der Gurnbergmoräne im zentralen Teil des Lainbachtales zentralalpine Blöcke in großer Zahl liegen (von der Spitze zum Hangfuß abnehmend, im Mittel $40 \%$, MuXFELDT 1972), sind sie im westlichen Teil im Bereich der Kohlstatt (zur Lage vgl. Abb. 2 ) selten $(<1 \%$ ). Ursache dieser räumlichen Differenzierung dürfte der Zustrom von Eismassen aus dem südlich anschließenden Pessenbachtal, das über eine Transfluenz mit dem Isargletscher verbunden war (DOBEN 1985), sein. Dieser Zweig des Isargletschers, der direkt aus dem Karwendelgebirge kam, war sicherlich sehr arm an Kristallingeschieben. In Aufschlüssen an der Kohlstatt fallen außerdem Radiolaritgeschiebe auf, die aus dem im Einzugsgebiet des Pessenbaches anstehenden Radiolarit stammen.

Im Gegensatz dazu weist der Isar-Walchenseegletscher und vor allem der Loisachgletscher im Raum Murnau einen höheren Kristallingeschiebegehalt mit bis zu $40 \%$ auf (DREESBACH 1985). Der größte Teil des Ferneises im Lainbachtal stammt daher aus dem Loisachgletscher bzw. dem Walchenseegletscher. Lediglich ein geringer Eiszustrom aus dem Pessenbachtal hat in der Zeit des Würmmaximums den Sattel (ca. $1050 \mathrm{~m}$ ü. NN) überschritten und zum Aufbau der Moränenwälle an der Kohlstatt beigetragen.

Die mächtigen Lockersedimentablagerungen, die im Untersuchungsgebiet an den Talflanken den anstehenden Fels überdecken, werden als $\mathrm{M}$ or ä n e n $\mathrm{s}$ e d i m e $\mathrm{n}$ t e des Ferneises angesprochen, da sie überwiegend aus kantigen Geschieben bestehen, kristalline Blöcke enthalten sowie nur an wenigen Stellen eine Schichtung erkennen lassen. Aufgrund von Eisbelastungen wurden sie stark verdichtet (BADER 1985). Der im Vergleich zu den Wallmoränen hohe Ton- und Schluffanteil dieser Sedimente, der die Verdichtung begünstigt, wurde durch die Gletschertrübe, die auch aus den lokalen Vergletscherungen in Richtung auf das Ferneis floß, vergrößert. Die seismischen Geschwindigkeiten sind in den Sedimenten der Moränenwälle mit ca. $1.7 \mathrm{~km} / \mathrm{s}$ deutlich niedriger als im darunterliegenden Moränenanteil (bis zu $3.1 \mathrm{~km} / \mathrm{s}$ ).

Der obere Abschluß der Moränenablagerung wird im Lainbachtal verbreitet durch $V$ e $r$ e b $n$ u $n$ g e $n$ gebildet, die heute oft nur in kleinen Restflächen erhalten sind. In Abbildung 2 ist die Lage der Verebnungsreste dargestellt. Es zeigen sich mehrere Flächenniveaus in unterschiedlichen Höhenlagen. Das höchste Niveau liegt zwischen 990 und $1020 \mathrm{~m}$ ü. NN und ist mit diesen Höhen auf die großen Moränenwälle, die sich besonders im südlichen Bereich des
Lainbachtales gut erhalten haben, eingestellt. Sie erreichen eine Höhe von 1030-1060 m ü. NN. Kurzzeitig muß allerdings das Ferneis über diese Wälle hinaus vorgestoßen sein, da sich im Oberlauf der Schmiedlaine Kristallingeschiebe noch in einer Entfernung von mehr als $1200 \mathrm{~m}$ südlich dieser Wälle nachweisen lassen. Moränenwälle dieses weitesten Vorstoßes sind nicht erhalten. Es lassen sich lediglich Hangleisten erkennen.

Das sich anschließende tiefere Niveau (Abb. 3) liegt zwischen 960 und $980 \mathrm{~m}$ ü. NN und korrespondiert mit den Resten eines Moränenwalles, der eine Höhe von $990-1000 \mathrm{~m}$ ü. NN erreicht.

Somit erweist sich auch die Vorstellung einer Abdachung der Oberkante der "Stausedimente" von $1020 \mathrm{~m}$ ü. NN im S des Lainbachtales auf $970 \mathrm{~m}$ ü. NN im N (BADER 1985) als nicht gegeben, da zwei unterschiedliche Verebnungsniveaus vorliegen.

Tiefer liegende Verebnungsniveaus bildeten sich während des Abschmelzens der Eismassen und markieren jeweils kurze Vorstoß- bzw. Stagnationsphasen des Gletschers. Die heute noch vorhandenen Reste dieser Verebnungen sind in Abbildung 2 wiedergegeben. Es finden sich mit abnehmender Höhe in Richtung Talausgang Verflachungen in $930 \mathrm{~m}$ ü. NN, $870 \mathrm{~m}$ ü. NN, $800 \mathrm{~m}$ ü. NN und $770 \mathrm{~m}$ ü. NN, die an vielen Lokalitäten belegt sind. Die eingezeichneten Flächen wurden im Gelände nachgewiesen und sind im Höhenlinienbild des Kartenblattes Kochel NO im Maßstab 1:10000 des Bayerischen Landesvermessungsamtes erkennbar.

Darüber hinaus gibt es singuläre Verebnungen, die auf einzelne, in dem stark gekammerten Relief zurückgebliebene Toteismassen bezogen sein können. Die nachweisbaren, hochgelegenen älteren Niveaus in $970 \mathrm{~m}$ ü. NN bzw. $1010 \mathrm{~m}$ ü. NN sind an ihrer Oberfläche durch ausgedehnte Ebenheiten gekennzeichnet. Die Verebnung ist nur schwer mit den oben dargelegten Vorstellungen zur Genese vereinbar, da die Moränenablagerungen des Ferneises (Ober-, Innen-, Grundmoräne, überfahrene und gestauchte, glazilimnische Ablagerungen am Eisrand) eine wellige Oberfläche aufweisen müßten. In der Vergangenheit kam man daher zu der Vorstellung der Sedimentation in einen Stausee. Während der Geländeaufnahmen zeigte sich, daß auf den Moränensedimenten zumeist wenige Meter mächtige Deckschichten feinkörniger, geschichteter Sedimente aufsitzen. Es ergibt sich daraus folgende $\mathrm{G}$ e n e s e d e r V e r e b. n u n g e n :

1. Vorstoß des Ferneises: Vor dem Gletscher abgelagerte Sedimente werden durch den vorrückenden 

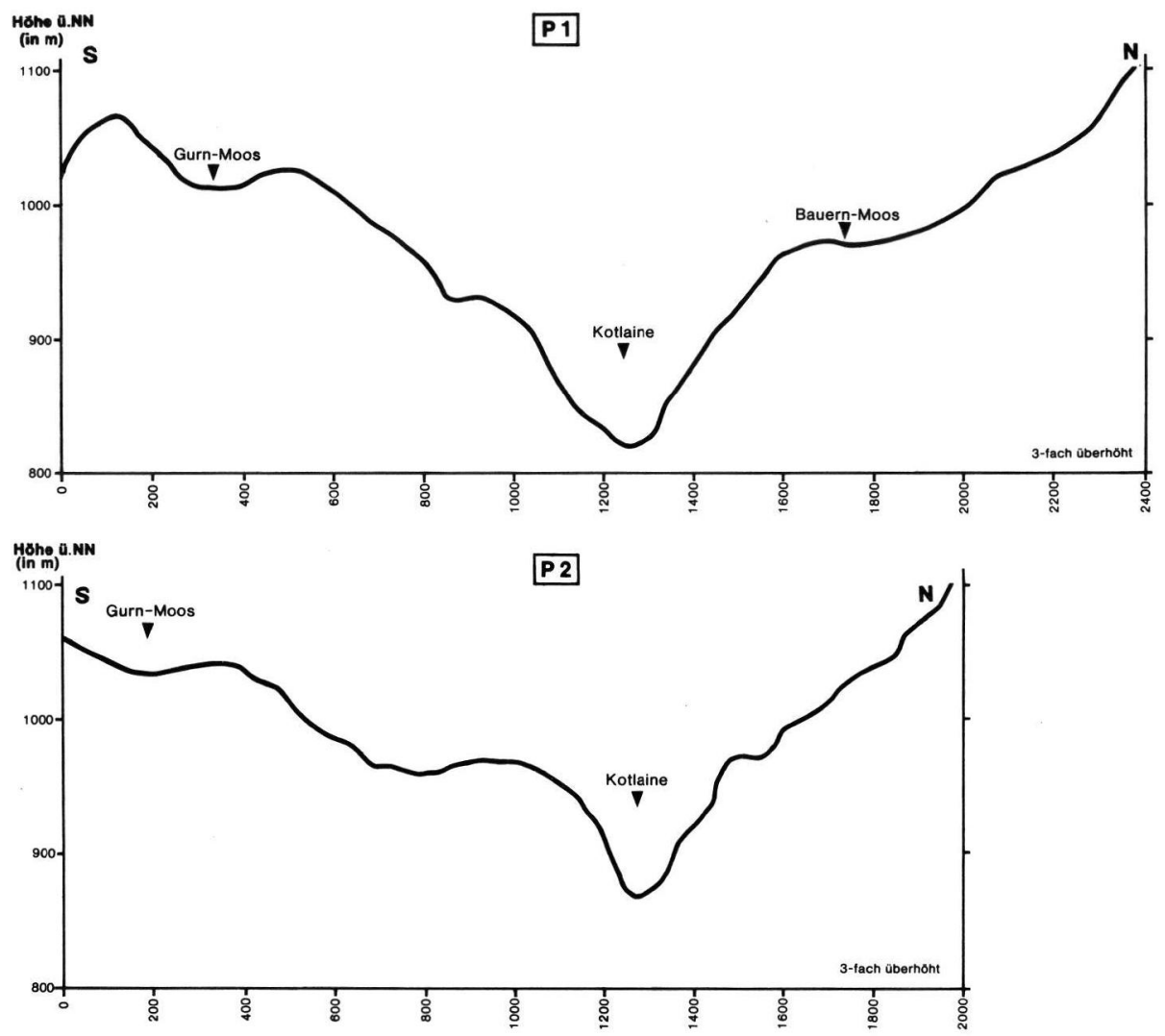

Abb. 3: Querprofildarstellungen der Verebnungen im Lainbachgebiet zwischen Bauernalm und Gurnberg (zur Lage vgl. Abb. 2).

Fig. 3: Transverse profiles of the planation surfaces in the Lainbach valley between the Bauernalm and the Gurnberg (s. Fig. 2).

Gletscher überfahren. Vorstoßschotter können sich bei vorherrschendem starken Gegengefälle nicht bilden. An einigen Stellen ist die Ausbildung von Umfliessungsrinnen möglich ( $z$. B. am Gurnberg zwischen den Moränenwällen).

2. Abschmelzen des Gletschers: Während dieser Phase werden die Moränensedimente ausschmelzen und zusammen mit den überfahrenen glazilimnischen Ablagerungen am Eisrand eine wellige Oberflächenstruktur zurücklassen.

3. Erneuter Gletschervorstoß oder Gletscherhalt: Vor dem Gletscher bilden sich Sedimentationsbecken, in denen feinkörnige, geschichtete Sedimente auf der Moränenoberfläche abgelagert werden, so daß diese eine Einebnung erfährt. Der Ton- und Schluffgehalt dieser Ablagerungen kann $90 \%-100 \%$ erreichen. Der Zyklus muß sich im Untersuchungsgebiet mehrfach wiederholt haben, weil auf den Moränensedimenten Verebnungn in unterschiedlichen Höhenniveus liegen (Abb. 2). Die schluffig-tonigen Ablage- rungen der Verebnungen weisen zum Teil warvenartige Schichtungen auf. Sie treten nur an wenigen Stellen mit Mächtigkeiten über drei Meter auf. Größere Mächtigkeiten können unter Beteiligung von Toteis entstanden sein, dessen Existenz durch einzelne Hohlformen heute noch dokumentiert werden kann (Abb. 2).

Die Genese dieser feinkörnigen Sedimente ist an lokale Sedimentationsräume gebunden, da kleinräumig wechselnde Deltaschüttungen zu erkennen sind und die Höhenlagen der Verebnungen oft auf einer Distanz von wenigen $100 \mathrm{~m}$ um mehr als $10 \mathrm{~m}$ divergieren. Ebenso lokal haben sich auf diesen schluffigtonigen Ablagerungen $\mathrm{M}$ o o r e gebildet.

Die Verebnungsniveaus sind mit den taleinwärts folgenden höher gelegenen Moränenwällen durch ansteigende Flächen verbunden (vgl. Abb. 3). Die ebene, kaum zertalte Oberläche dieser Hänge könnte im eisrandnahen Bereich unter den periglazialen Klimabedingungen ausgebildet worden sein. 


\subsection{Der Aufbau des Moränenkörpers}

Die mächtigen Moränensedimente zwischen den Moränenwällen im Bereich des Gurnmooses wurden von zwei Erkundungsbohrungen (vgl. Abb. 2), die im Auftrag des Wasserwirtschaftsamtes in Weilheim durchgeführt und als Grundwasserpegel ausgebaut wurden, erschlossen. Zusätzlich stehen die Ergebnisse der geoseismischen Aufnahme (BADER 1985) sowie Messungen der Strömungsrichtung und -geschwindigkeit in den Grundwasserhorizonten, die vom Institut für Hydrologie der Gesellschaft für Strahlen- und Umweltforschung (GSF) mit der Einbohrlochmethode an den Pegeln durchgeführt wurden, zur Verfügung. Die Ergebnisse können wie folgt zusammengefaßt werden:

1. Die bis in eine Tiefe von ca. $60 \mathrm{~m}$ gekernten Bohrungen zeigen, daß keine makroskopisch sichtbare Schichtung auftritt. Die Kornzusammensetzung schwankt, da der Kies- und Blockanteil stark variiert. Im Mittel der Bohrungen liegt der Sand-, Kies- und Blockgehalt insgesamt bei etwa $55 \%$. Der Ton- und Schluffgehalt erreicht mit $45 \%$ etwas höhere Werte als bei MuXFELDT (1972), der für den nahegelegenen Bereich der Melcherreiße einen mittleren Ton- und Schluffgehalt von $34 \%$ angibt. Es ist hier zu vermuten, daß das Feinkorn in den von ihm erfaßten oberflächlich anstehenden Lockersedimenten schon etwas ausgespült wurde und daher zurücktritt.
2. Die geoseismischen Messungen zeigen, daß die Moränensedimente hier zweigeteilt sind. Bis etwa $45 \mathrm{~m}$ unter Flur wurden dichte, darunter sogar sehr dichte Sedimente registriert (BADER 1985).

3. Das Grundwasser fließt im oberen Bereich nach W bzw. NW. In tiefer liegenden Horizonten wurde eine reine Nordströmung festgestellt, die aufgrund der höheren Dichte der Sedimente deutlich langsamer fließt (BECHT \& KOPP 1988).

Die Ursache der seismisch und hydrologisch nachgewiesenen Zweiteilung des Moränenkörpers ist in der Genese zu suchen. Die Auswertung der seismischen Untersuchungen ergab an der Stelle der Bohrungen im tieferen Untergrund (ca. $150 \mathrm{~m}$ unter Geländeoberkante) ein im anstehenden Fels ausgebildetes Kerbtal, das mit den Lockersedimenten verfüllt wurde (Abb. 4). Bei Beginn des Eisvorstoßes folgt der Gletscher zunächst diesem Kerbtal und stieß von Norden aus dem Lainbachtal gegen die Benediktenwand vor. Da sich der Gletscher gegen das Gefälle des Untergrundes vorschob, konnten sich in den Moränensedimenten nach Norden ausgerichtete Strukturen bilden, die heute makroskopisch in den Erosionsformen nur selten erkennbar sind. Mit dem Anwachsen der Eismächtigkeit zum Würmmaximum stieß der Gletscher von NW bis W in das Tal vor. Die Fließrichtung der heutigen Grundwasserströme im oberen Bereich der Lockersedimente folgt also den sich ändernden Sedimentationsrichtungen des Moränenmaterials.

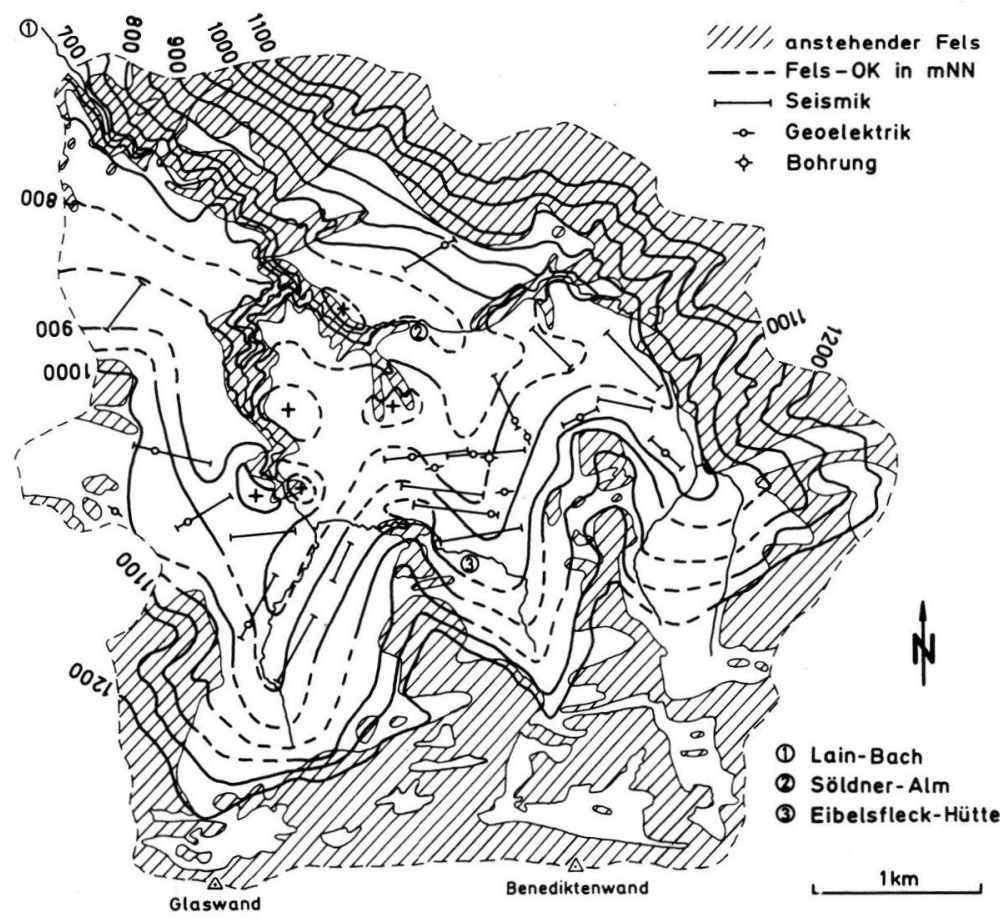

Abb. 4: Felsoberfläche im Bereich der

Lainbach-Staubeckensedimente südöstlich Benediktbeuern

(BADER 1985: 75).

Fig. 4: The rock surface in the region of the pleistocene loose sediments in the Lainbach valley south-eastern Benediktbeuern. 
Zeitweise kann sich zwischen den beiden Moränenwällen auch eine in Richtung W entwässernde Umfließungsrinne ausgebildet haben (vgl. 2.2).

Pollenanalytische Untersuchungen an den Bohrkernen der Erkundungsbohrungen zeigten, daß unter einer würmzeitlichen Moränenauflage „Ablagerungen mindestens zweier Interglaziale und zweier ausgeprägter Kaltzeiten folgen" (FRENZEL 1987: 171). Das Kerbtalrelief im Anstehenden ist danach spätestens im Mittelpleistozän (Mindel) verschüttet worden. Mehrfach sich wiederholende glaziale Verschüttungen und interglaziale Erosionsphasen, die nur einen Teil der Locksersedimente erfaßten, schufen einen komplex aufgebauten Sedimentkörper, der makroskopisch erstaunlich homogen erscheint.

\subsection{Spätglaziale Seeablagerungen}

Das unterste Verebnungsniveau auf $750 \mathrm{~m}$ ü. NN (vgl. 2.2 und Abb. 2) schließt im Gegensatz zu den darüber liegenden Flächenresten wiederum an einen Moränenwall an. Er liegt, wie aus Abbildung 2 hervorgeht, vor dem Tal und markiert somit einen späten Vorstoß des Walchensee- und Loisachgletschers, der allerdings nur noch eine geringe Mächtigkeit besaß. Die Moränenwälle liegen bei 770-785 m ü. NN. In dieser Situation konnte sich ein kleiner Rückstau der Abflüsse aus dem Einzugsgebiet des Lainbaches bilden. Die Sedimente dieses Stauraumes findet man als tonig-schluffige $z$. T. mit sandigen Einlagen durchsetzte Horizonte, die zwischen 740 und $760 \mathrm{~m}$ ü. NN in den Bächen an den Talflanken des Lainbaches aufgeschlossen sind. Die Entstehung dieses Stauraumes und somit auch dessen Ablagerungen setzt voraus, daß der Gletscher im Lainbachtal weitgehend abgeschmolzen war und mögliche Moränenreste zumindest im Unterlauf im Spätglazial schon großteils ausgeräumt waren. Daraus folgt, daß es eine frühholozäne kräftige Erosion, wie sie von KARL \& DANZ (1969) vermutet wurde (vgl. Abb. 1), nicht gegeben hat, sondern daß die kräftige Erosion an den Hängen schon mit dem Abschmelzen des Gletscherlobus im Tal einsetzte.

Eine Ansprache der tonig-schluffigen Sedimente in $750 \mathrm{~m}$ ü. NN als „Basistone” scheidet aus, da die Entstehung dieser Ablagerungen nicht mit einem frühwürmzeitlichen Vorstoß des Isar-Walchensee oder des Loisachgletschers in Verbindung steht. An anderen, höher gelegenen Lokalitäten finden sich im Lainbachtal keine tonig-schluffigen Sedimente an der Basis der Moränensedimente. Es ist nicht auszuschließen, daß mit beginnendem Eisvorstoß Sedimentationsbecken, die dem späten Gletschervorstoß in der Dimension entsprachen, entstanden. Mit dem Vorstoß des Fern- eises in das Lainbachtal wurden diese Sedimente wieder aufgenommen und sind daher heute nicht mehr nachweisbar.

\section{Spät- und postglaziale Erosion der Lockersedimente im Lainbachtal}

Die aktuelle Erosionsleistung im Einzugsgebiet des Lainbaches kann mit 20000 t/a Feststoffaustrag angesetzt werden (BECHT 1986). Geht man davon aus, daß etwa $75 \%$ der Sedimente aus den pleistozänen Ablagerungen stammen, dann ergibt sich bei einem spezifischen Gewicht des Substrates von $2500 \mathrm{~kg} / \mathrm{cbm}$ ein Betrag von ca. $6000 \mathrm{cbm} / \mathrm{a}$. Würde man diese Summe auf 15000 Jahre spät- und postglazialer Erosionszeit hochrechnen, ergibt sich ein Wert von 90 Mio cbm. Obwohl dieser Betrag in der Größenordnung und nur die sollte hier betrachtet werden - recht gut mit den im Schwemmfächer des Lainbaches lagernden ca. 100 Mio cbm Ausraummasse übereinstimmt, kann nicht von einer kontinuierlich verlaufenden Erosion in diesem Zeitraum ausgegangen werden.

Die Auswertung des vorliegenden Kartenmaterials sowie der Luftbilder ab 1959 (BECHT \& KopP 1988) ergab, daß die Erosion in der zweiten Hälfte des 19. Jahrhunderts sowie zu Beginn des 20. Jahrhunderts erheblich stärker war als heute. Berichte von Vermurungen und katastrophalen Hochwasserereignissen aus dem späten Mittelalter (DAFFNER 1883) lassen vermuten, daß landwirtschaftliche Nutzung des Lainbachgebietes, die aufgrund von Pollenanalysen durch FRENZEL und BLUDAU (Universität Hohenheim) an zahlreichen Mooren für das Mittelalter nachgewiesen wurde, verbreitet zu Erosionsschäden geführt hat. Die Waldweide an Erosionshängen wurde erst 1957 aufgegeben (BECHT \& KopP 1988). Neben diesen spätmittelalterlichen und neuzeitlichen Erosionsphasen zeugen alte, heute unter Wald liegende Anrißformen von früherer intensiver Abtragung. Eine zeitliche Einordnung kann bisher nicht erfolgen. Es könnte sich um spätglaziale Erosion unmittelbar vor dem Gletscher handeln. Dafür spräche auch die Ausrichtung der Längsprofile dieser alten Anrisse auf ein deutlich über dem heutigen Vorfluter liegendes Niveau, das durch stagnierendes Eis gebildet worden sein könnte. Eine rasche Wiederbegrünung der Hänge noch unter den gleichen Klimabedingungen hat sicher zu einem schnellen Rückgang der Erosionsleistung beigetragen. $\mathrm{Ob}$ es Zeiten der Formungsruhe gab, läßt sich heute nicht entscheiden. Vielleicht rissen auch ohne Eingriffe des Menschen, die schon für die Bronzezeit aufgrund der Pollenanalysen nachgewiesen werden können (FRENZEL 1987), immer wieder kleine Erosionsherde auf, die dann natürlich wiederbegrünten. Man kann aber davon ausgehen, daß seit dem Mittelalter 
mit der wirtschaftlichen Nutzung der Bergwälder (Weide, Holzkohlegewinnung, Brennholzeinschlag, kleinräumig auch Ackerbau) die Erosion stark zunahm und z. T. sicherlich kräftiger war als heute.

\section{Schlußbemerkung}

Die Genese pleistozäner Lockersedimente im Lainbachtal konnte auf den dominanten Einfluß des Loisach- und Isar-Walchenseegletschers, der bis weit in das Tal vorstieß, zurückgeführt werden. Diese Deutung der Entstehung der Sedimente im Lainbachgebiet läßt sich nicht auf jedes randalpine Seitental übertragen. Eine Prüfung im Einzelfall muß den Nachweis erbringen, wie die Lockersedimente transportiert und abgelagert wurden. So zeigt sich beispielsweise im Halblechgebiet bei Füssen ein Nebeneinander von glazialer und fluvialer Formung:

Während der Lechgletscher im Haupttal des Halblechs vorstieß und Moränenablagerungen hinterließ, ist im oberen Lobenbachtal, das von $\mathrm{S}$ in das Halblechtal mündet, eine ausgeprägte, mehrere Zehner Meter mächtige Folge von deltaartig geschichteten Sedimenten aufgeschlossen. Der Lechgletscher erfaßte vom Halblechtal ausgehend nurmehr den unteren Teil des Lobenbachtales mit seinen Moränen. In dem engen, oberen Bereich des Tales, das gegen die Bewegungsrichtung des Eises im Lechtal durchströmt werden müßte, konnte das Ferneis nicht vorstoßen. Das Beispiel zeigt, daß die Genese der pleistozänen Lockersedimente stark an die lokalen Reliefbedingungen geknüpft ist. Die glaziale Formung ist dabei zunächst als Regelfall anzusehen, die fluviale Sedimentation in Stauseen dagegen die Ausnahme.

\section{Schriftenverzeichnis}

BADER, K. (1979): Exarationstiefen würmzeitlicher und älterer Gletscher in Südbayern (Trennung eisvorbelasteter und nicht eisvorbelasteter Sedimente aufgrund seismischer Geschwindigkeiten). - Eiszeitalter u. Gegenwart, 29: 49-61; Hannover.
BADER, K. (1985): Geophysikalische Untersuchungen. In. K. DoBEN: Geologische Karte von Bayern 1:25 000, Erläuterungen zum Blatt Nr. 8334 Kochel a. See. 134 S., 20 Abb., 2 Tab.; München.

BECHT, M. (1986): Die Schwebstofführung der Gewässer im Lainbachtal bei Benediktbeuern/Obb. - Münchener Geogr. Abh., B2, Reihe B, 201 S., 110 Abb., 13 Tab.; München.

— \& KopP, M. (1988): Aktuelle Geomorphodynamik in einem randalpinen Wildbacheinzugsgebiet und deren Beeinflussung durch die Wirtschaftsweise des Menschen. - Veröff. 46. Deutschen Geographentag in München 1987: 526-534, 5 Abb., Stuttgart.

DAFFNER, F. (1893): Geschichte des Klosters Benediktbeuern. - Literarisches Inst. Dr. M. Hutter, 432 S., 2 Bl., München.

DOBEN, K. (1985): Geologische Karte von Bayern $1: 25000$, Erläuterungen zum Blatt Nr. 8334 Kochel am See. 134 S., 20 Abb., 2 Tab.; München (Bayer. Geol. L. -Amt).

DreEsBACH, R. (1986): Sedimentpetrographische Untersuchungen zur Stratigraphie des Würmglazials im Bereich des Isar-Loisachgletschers. - Diss. Universität München, 25 Tab., 29 Abb., 176 S.; München.

FRENZEL, B. (1987): Forschungen zur Geographie und Geschichte des Eiszeitalters (Pleistozän) und der Nacheiszeit (Holozän). - Jb. d. Akad. d. Wiss. u. d. Lit.: 169-176; Mainz.

KARL, J. \& DANZ, W. (1969): Der Einfluß des Menschen auf die Erosion im Bergland. - Schrift.-Reihe der Bayer. Landesstelle f. Gewässerkunde, 1: 98 S., 32 Abb., 17 Karten, München.

Mưller-Deile, G. (1940): Geologie der Alpenrandzone beiderseits vom Kochelsee in Oberbayern. - Mitt. Reichsanst. Bodenforsch., 34: 109 S., 32 Abb., 9 Taf.; München.

MuxfeldT, J. (1972): Der Aufbau der pleistozänen Talverschüttung im Bereich der Kotlaine. - Zulassungsarb. LA Gymn.: 19 S., 13 Abb., 2 Tab.; München. [Unveröff.].

Manuskript eingegangen am 5. 12. 88,

Nachträge April 1989. 